\title{
High resolution spectroscopy of HD 207538 from Far-UV (FUSE) to Visible (SARG-TNG)
}

\section{A global picture of the stellar and interstellar features modeled}

\author{
G. Catanzaro ${ }^{1}$, M. K. André ${ }^{2}$, F. Leone ${ }^{1}$, and P. Sonnentrucker ${ }^{3}$ \\ 1 INAF - Catania Astrophysical Observatory, Via S. Sofia 78, 95123 Catania, Italy \\ e-mail: fleone@ct.astro.it \\ 2 Institut d'Astrophysique de Paris, CNRS, 98bis boulevard Arago, 75014 Paris, France \\ e-mail: andre@iap.fr \\ 3 The Johns Hopkins University, Center for Astrophysical Sciences, 3400 N. Charles St., Baltimore, MD 21218-2695, USA \\ e-mail: sonnentr@pha.jhu.edu
}

Received 20 December 2002 / Accepted 31 March 2003

\begin{abstract}
We present a detailed study of the suspected Chemically Peculiar star HD 207538 based on high resolution spectroscopy in the far Ultraviolet (1000-3350 $)$ and visible (4600-7000 $\AA$ ) ranges. The stellar abundance synthesis analysis was performed by using Kurucz's codes ATLAS9 and SYNTHE to compute the atmospheric model and the synthetic spectrum respectively.

Observations were obtained with the Far Ultraviolet Spectroscopic Explorer, the International Ultraviolet Explorer satellites whereas the optical spectrum was collected with the ground-based Telescopio Nazionale Galileo telescope.

With our analysis we refine the value of the rotational velocity to $v_{\mathrm{e}} \sin i=42 \pm 3 \mathrm{~km} \mathrm{~s}^{-1}$ and the microturbulence velocity to $\xi=8 \pm 1 \mathrm{~km} \mathrm{~s}^{-1}$. The stellar abundances inferred in this study show that $\mathrm{C}, \mathrm{N}, \mathrm{O}, \mathrm{Al}, \mathrm{Si}, \mathrm{P}, \mathrm{S}$ and $\mathrm{Mn}$ are compatible with the standard solar abundances, within the experimental errors. On the other hand, $\mathrm{Fe}, \mathrm{Ni}$ and $\mathrm{Zn}$ are definitively underabundant. We also show that the helium content is comparable to the typical abundance of B-type stars.

Because this spectrum is rich in stellar and interstellar features, we computed simultaneously a synthetic spectrum of the stellar and interstellar lines in order to disentangle the different absorption contributions. We thus also determined the column densities of several interstellar elements (FeII, NI, PII, HI, $\mathrm{H}_{2}, \mathrm{HD}, \mathrm{CO}, \mathrm{ClI}$ and ArI) present in the gas in front of the star. Although several components are present along this sightline, our results suggest the presence of some translucent interstellar gas among the diffuse components.

With this work, we demonstrate that it is possible to perform a stellar spectroscopic analysis from the far-UV to visible spectral range, with consistent results in both ranges within errors, based on one single atmosphere model.
\end{abstract}

Key words. stars: individual: HD 207538 - stars: abundances - stars: atmospheres - ultraviolet: stars - ISM: abundances ISM: lines and bands

\section{Introduction}

The most commonly used code to compute stellar atmosphere models is ATLAS (Kurucz 1993). These models correctly reproduce the observed flux distribution from the UV to the visible range and match the Balmer line profiles not only for solar composition stars (Castelli \& Kurucz 1994) but also for magnetic Chemically Peculiar (CP) stars (Leone \& Manfrè 1996).

Recently ATLAS models have been also used to interpret the far-UV spectrum of stars. However, several authors (i.e. Chavez et al. 1995; Buss et al. 1995; Morales et al. 2001;

Send offprint requests to: G. Catanzaro,

e-mail: gca@ct.astro.it
Frémat et al. 2002) concluded that the Kurucz LTE atmospheric models fail in reproducing the temperature distribution of the outermost layers. In particular, these models seem to underestimate the stellar fluxes between $\mathrm{Ly}_{\alpha}$ and $\mathrm{Ly}_{\beta}$. Dixon \& Hurwitz (1998) noted this behavior in their model and suggested that these discrepancies may be due to abundance anomalies or nonLTE effects.

Within our program dedicated to the study of CP stars carried out with high resolution spectroscopy, we have observed the suspected CP star HD 207538 (Renson 1991). This star was also observed with the International Ultraviolet Explorer (IUE) and the Far Ultraviolet Spectroscopic Explorer (FUSE) satellites. By combining these archive far-UV data with our optical 
spectra, we test the capability of Kurucz LTE codes to model the observed spectra of HD 207538 from the far-UV to visible range with a single set of atmosphere stellar parameters.

In the spectral range covered by FUSE, (912-1188 ̊), the contamination due to the interstellar lines is important. We thus also performed a detailed study of the interstellar medium in order to obtain a global synthetic model in the far-UV combining both stellar and interstellar models. In Sect. 2, we summarize the results obtained prior to this work on the CP star HD 207538. Section 3 describes the observations and data reductions. The results of the stellar and interstellar analyses are given in Sects. 4 and 5, respectively. Our concluding remarks are given in Sect. 6.

\section{HD 207538}

HD 207538 is a B0V star belonging to the Cep OB2 association at $615 \pm 35 \mathrm{pc}$ from the Sun (De Zeeuw et al. 1999). Recent studies on the chemical abundances of metals in OB star members of Cep OB2 led Daflon et al. $(1999,2001)$ to conclude that the chemical composition in the association is homogeneous and underabundant with respect to the Sun by roughly 0.3 dex. In the specific case of HD 207538, they also concluded that the $\mathrm{N}, \mathrm{O}$ and $\mathrm{Si}$ abundances are similar to the solar values.

HD 207538 is reported in the General Catalogue of Ap and Am stars (Renson 1991) as a CP candidate star with helium overabundance. The main observational characteristics of $\mathrm{CP}$ stars are the spectral, photometric and magnetic variations with a common period. According to the Oblique Rotator Model (Stibbs 1950) this period is simply the rotational period and variations are due to the non-homogeneous distribution of elements on the stellar surface.

The helium peculiar stars are known to show not only spectral variability but also photometric variability (Peterson 1966). However, Vetö et al. (1980) found that HD 207538 shows no periodic light variability. Our own investigation of the Hipparcos photometric data (spanning 1132 days), using the Phase Dispersion Method (Stellingwerf 1978) as coded in the NOAO/IRAF package, does not indicate any evidence of variability either. Therefore, the peculiar nature of this star is not yet firmly established photometrically.

\section{Observations and data reduction}

The spectra analyzed in this paper have been acquired with the following instruments depending on the wavelength range.

\subsection{Optical data $(\lambda \lambda 4600-7000 \AA)$}

On August 10, 2000, we obtained an echelle spectrum of HD 207538 with the high resolution spectrograph (SARG) (Gratton et al. 2003) and the $3.55 \mathrm{~m}$ Telescopio Nazionale Galileo (TNG) at the Observatorio del Roque de los Muchachos (La Palma, Spain).

Covering the region from 4600 to $7000 \AA$, our stellar spectrum calibrated in wavelength and normalized in flux, was obtained using standard reduction procedures for spectroscopic observations within the IRAF package. The calibration lamp lines show that the achieved resolving power is $R=115000$ and the $S / N$ ratio is between 60 and 100 .

Equivalent widths have been measured with a Gaussian fit of each line using standard IRAF routines. As the main source of errors on the equivalent width measurements is the uncertain position of the continuum, we estimated the error to be equal to half the area of a box as wide as the line and with a height equal to rms of the continuum.

In order to check the possible spectral variability, on October 19, 2002, we obtained a second spectrum of HD 207538 with the $91 \mathrm{~cm}$ telescope equipped with the REOSC echelle spectrograph of the Catania Astrophysical Observatory. The region covered by our spectrum is 4820 $5670 \AA$ at $R=13000$ resolution. Data reduction was also performed by means of IRAF package, and the final normalized spectrum shows that the $S / N$ ratio was between 100-150.

\subsection{IUE spectral range $(\lambda \lambda 1150-3350 \AA)$}

The IUE spectra of HD 207538 processed with the NEWSIPS reduction method have been taken from the INES Final Archive data. The images consist of four high dispersion spectra, two of which have been obtained with the Short-Wavelength Prime (SWP: 1150-2000 A) while the two others have been obtained with the Long-Wavelength Redundant (LWR: 1850-3000 ̊) cameras (see Table 1 and Nichols \& Linsky 1996).

After an accurate check of the flux constancy, we manually coadded all these observations obtaining a unique spectrum which covers the $1150-3350 \AA$ interval. The resulting $S / N$ ratio was about 20 . No evidence of the flux variability characterizing CP stars (Leckrone 1974) was found.

\subsection{Far UV data $(\lambda \lambda \quad 1000-1188 \AA)$}

The FUSE satellite and the spectrograph mounted on board were described by Moos et al. (2000) and Sahnow et al. (2000). HD 207538 was observed in time-tag mode on 8th December 1999. This early-type star, shining through interstellar clouds with a total reddening $E_{(B-V)}=0.64$ and a total extinction $A_{v}=1.43$ (Snow et al. 2002), is bright enough to allow a good $S / N$ ratio. The foreground dust and gas show large column densities of many atomic and molecular species. The FUSE spectra were extracted from 4 sub-exposures totalizing $7736 \mathrm{~s}$ using the large aperture (LWRS) with the CALFUSE (Version 1.8.4) standard reduction pipeline which provided us with flux and wavelength calibrated spectra.

Table 1. Journal of observations concerning the IUE spectra used in this study.

\begin{tabular}{ccccc}
\hline \hline Image & Disp. & Date & UT & Exp (s) \\
\hline SWP08992 & High & $05 / 14 / 1980$ & $14: 16: 14$ & 3900 \\
SWP09872 & High & $08 / 23 / 1980$ & $00: 41: 22$ & 4020 \\
LWR07750 & High & $05 / 14 / 1980$ & $13: 27: 15$ & 1500 \\
LWR07751 & High & $05 / 14 / 1980$ & $15: 25: 35$ & 2520 \\
\hline
\end{tabular}



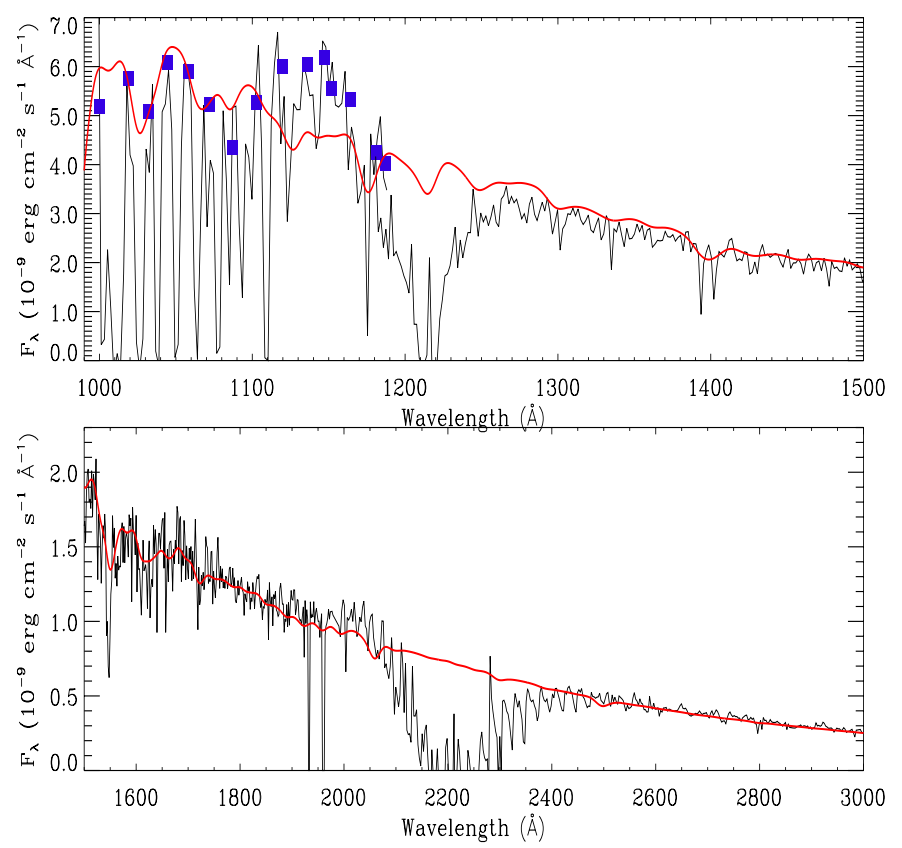

Fig. 1. Observed (thick line) and computed (thin line, $R=100$ ) flux distribution of HD 207538. The ATLAS9 atmosphere model has been computed assuming $T_{\text {eff }}=32190 \mathrm{~K}, \log g=4.32$, solar metal opacity and microturbulence velocity equal to $8.0 \mathrm{~km} \mathrm{~s}^{-1}$. Because of the presence of large absorption due to the interstellar medium, FUSE data are averaged within $2 \AA$-width bands centered at the highest values of the flux distribution in order to visualize the differences between the observed and computed spectra (filled squares). The extinction bump at $\lambda 2175 \AA$ is clearly visible on this plot.

The processing steps included data screening, thermal drift correction, geometric distortion correction, Doppler correction to heliocentric wavelength, dead time correction and wavelength calibration. The resolving power was $R=15000$. These spectra were then coadded and binned over 4 pixels (less than one resolution element), using both exposure time and error weighting, to obtain a $S / N$ ratio of 15 per resolution element. Strong interstellar extinction and misalignment of the SiC channels prevented us, however, from using data shortward of $1000 \AA$.

\section{Stellar spectrum analysis}

Usually, spectral synthesis is performed assuming a precomputed atmosphere model whose parameters are often fixed from photometric indices. In a previous paper (Leone \& Manfrè 1996), we have discussed the importance of consistency between the parameters adopted for computing the atmosphere model and the determined parameters. As to CP stars, a correct metal opacity is fundamental in determining the stellar effective temperature and gravity. In the case of a hot star, like HD 207538, particular attention has to be payed to the microturbulence. Large values for this velocity can significantly desaturate metal lines and increase the metal opacity with respect to a more static atmosphere.

Several steps are necessary to determine the stellar parameters such as the effective temperature, gravity, abundances and microturbulence. In our analysis, following the method described in Leone \& Manfrè (1996), we have used an iterative process to determine the stellar parameters.

\subsection{Atmosphere model}

To compute the stellar atmosphere model of HD 207538, we used version 9 of ATLAS (Kurucz 1993). This code includes the metal opacity by means of distribution functions that are tabulated for multiples of the solar metallicity and for various microturbulent velocities. So that, metal opacity scale and microturbulence velocity adopted to compute the atmosphere model have to be consistent with the derived abundances, measured microturbulence, effective temperature and gravity.

Prior to the model calculation with ATLAS9, the atmosphere is divided up in a number of layers starting from a fixed Rosseland optical depth. Because of the very large spectral range we intend to model, a check that all identified spectral lines, in the far-UV and visible ranges, are formed within the adopted limits is performed. We chose to divide the atmosphere into the maximum (99) number of layers from $\log \tau_{\text {Ross }}=-4.8$ to 3.12 .

Abundances have been derived by means of WIDTH9 (Kurucz 1993) and the microturbulent velocity determined, imposing that the oxygen abundance is independent of the equivalent widths measured for seven OII of the visible range and three OIII of the far UV.

At the end of the iterative procedure, where the UV-Visible flux overall distribution and the $\mathrm{H}_{\gamma}$ wings were matched, we found that the effective temperature $\left(T_{\mathrm{eff}}=32190 \mathrm{~K}\right)$ and the gravity $(\log g=4.32)$ determined by Daflon et al. (1999) agree and can be adopted. In contrast we found $\xi=8 \pm 1 \mathrm{~km} \mathrm{~s}^{-1}$, a microturbulence velocity slightly smaller that the $10 \mathrm{~km} \mathrm{~s}^{-1}$ fixed by the same authors.

\subsection{UV flux distribution}

Using the adopted effective temperature and gravity we computed the synthetic spectrum that reproduces the observed UV flux distribution.

The synthetic spectrum was computed with SYNTHE (Kurucz \& Avrett 1981) from 1000 to $7000 \AA$ and converted to absolute flux, by means of the relation $\log F(5556 \AA)=$ $-0.4 V-8.451$ where $V=7.31$ is the magnitude quoted in the SIMBAD database.

Dereddening of the observed spectra has been performed following Cardelli et al. (1989) assuming $E_{(B-V)}=0.68$, a value not far from the 0.64 value given by Snow et al. (2002). Figure 1 shows the observed and computed spectra. We note that the computed spectrum represents well the IUE flux distribution and the FUSE spectrum for wavelengths shorter than $1100 \AA$. However, the FUSE flux for wavelengths longer than $1100 \AA$, is larger than the computed one. We found no combination of effective temperature, gravity and dereddening able to match the overall flux distribution from 900 to $3350 \AA$. In particular, the apparent flat flux distribution recorded by FUSE could not be reproduced. 


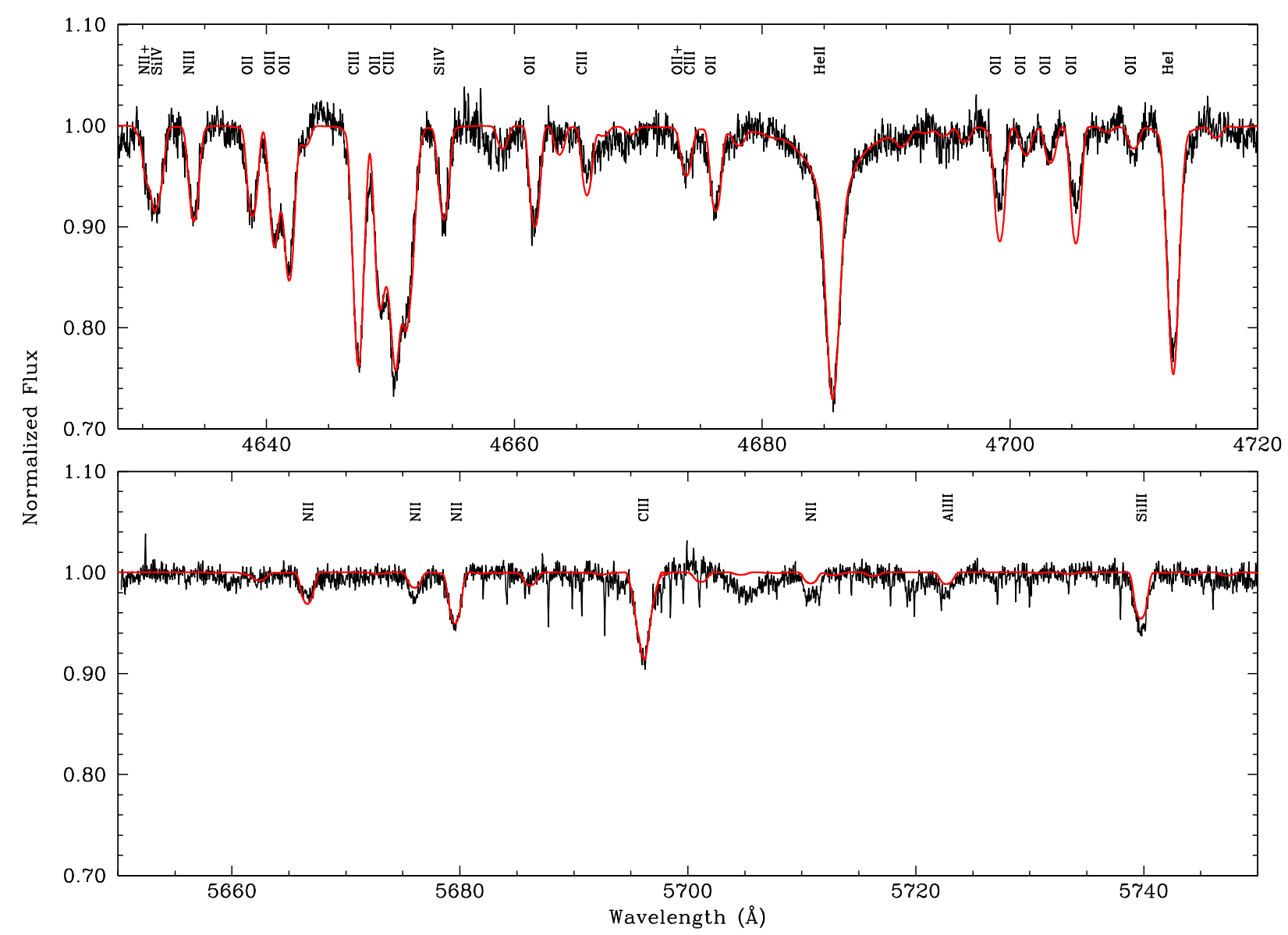

Fig. 2. Comparison between theoretical (smoothed line) and observed spectra in the visible range $\lambda \lambda 4625-4720 \AA$ (top panel) and $\lambda \lambda 5650-5750$ (bottom panel). In each panel we label the identified lines.

Differences between the observed and computed spectra are largest around $1150 \AA$, where they amount up to $20 \%$. It is tempting to conclude that ATLAS9 models are not able to reproduce FUSE data. However there are other possible causes that need to be addressed as well:

- the $S / N$ ratio of our spectrum is $\sim 15$, so the continuum is determined with an error of about $6 \%$;

- dereddening at the FUSE wavelengths is an extrapolation of a law defined only for wavelengths longer than $1250 \AA$;

- absolute calibration flux of FUSE data is based on modelatmosphere predictions of the spectra of well-studied white-dwarf stars (Sahnow et al. 2000) and is expected to be accurate at the $10 \%$ level. Moreover, we know that the instrument's sensitivity was nearly constant for the first two years of the mission and that it has later declined by about $10 \%$. So a further few percent uncertainty is expected in the flux calibration.

\subsection{SARG-TNG data}

A synthetic spectrum covering the observed optical range was calculated with SYNTHE (Kurucz \& Avrett 1981) and convolved with a Gaussian profile to match the instrumental resolution. To identify spectral lines and to derive abundances we adopted the atomic parameters from the Kurucz (1993) line list. The rotational velocity has been determined by matching the line profiles of three unblended OI lines (OI $\lambda \lambda 4638.861$, 4661.643 and $4676.244 \AA)$, one NIII line ( $\lambda 4634.126 \AA$ ) and one SiIV line $(\lambda 4654.312 \AA)$. The obtained value $v_{\mathrm{e}} \sin i=$ $42 \pm 3 \mathrm{~km} \mathrm{~s}^{-1}$ is slightly less than the value of $47 \mathrm{~km} \mathrm{~s}^{-1}$ inferred by Penny (1996) for HD 207538.

In Fig. 2, we report the comparison between the synthetic and observed spectrum for the richest spectral regions: the 4625-4720 $\AA$ and 5650-5750 $\AA$ windows containing a number of metallic lines due to $\mathrm{C}, \mathrm{N}, \mathrm{O}, \mathrm{Si}$ and $\mathrm{Al}$ at various stages of ionization. For comparison with the observations, the theoretical spectra was convolved with the instrumental profile and the rotational velocity.

With the exception of helium that we will discuss in the next subsection, solar standard abundances (Grevesse et al. 1996) are sufficient to reproduce all the metalic lines present in Fig. 2. Errors have been estimated to be 0.2 dex. Adopting the opacity distribution function for solar abundances to compute the atmosphere model is therefore justified.

\subsubsection{Helium lines}

In our optical spectrum of HD 207538, we identify nine unblended helium lines (two HeII and seven HeI), whose measured equivalent widths are listed in Table 2. These lines were previously measured by Conti \& Alschuler (1971 HeII $24686 \AA$ Å), Mathys (1989), Grigsby et al. (1992); other 

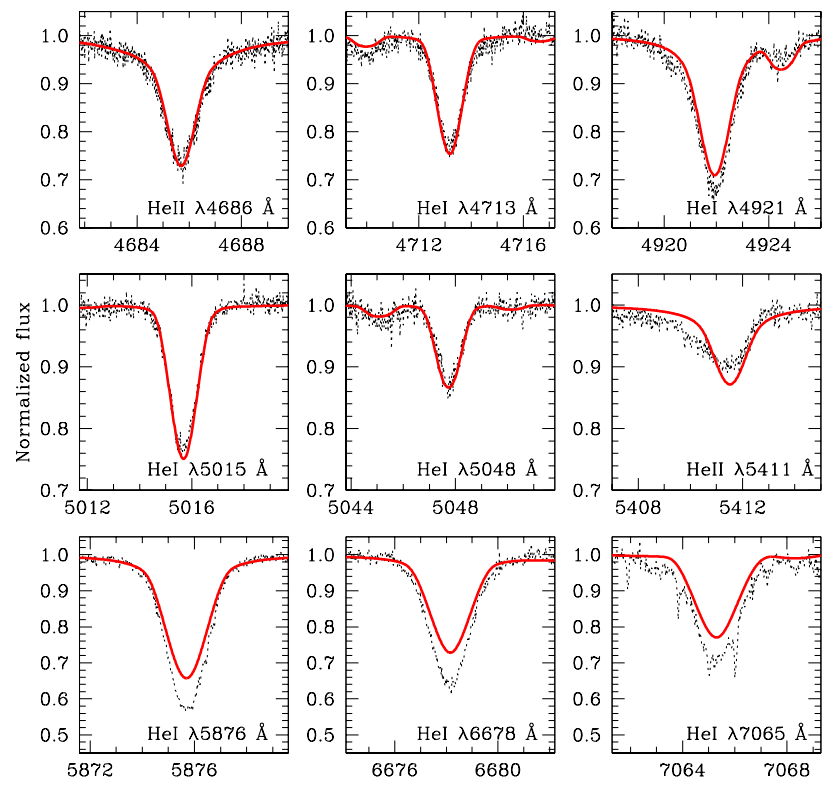

Wavelength $(\AA)$

Fig. 3. Helium lines observed (dotted line) in the visible spectrum of HD 207538. For each line we overplotted the corresponding synthetic profile (solid/red curve).

measurements have been carried out by Conti $(1973,1974)$ for the HeI $\lambda 5876 \AA$ and the HeI $\lambda 6678 \AA$. Within experimental errors, all these measurements are in agreement with ours.

As reported by Conti (1974), we confirm that the profile of HeII $\lambda 5411 \AA$ is strongly distorted by an asymmetry toward the blue wing of the line. This feature is still visible even in our low resolution spectrum taken at Catania Astrophysical Observatory.

Profiles of helium lines were computed in the LTE approximation for a variety of $\mathrm{He} / \mathrm{H}$ ratios with the adopted model atmosphere by using the program SYNTHE and a consistent ATLAS9 model. The abundance that best fits the He lines on average is $\log \epsilon(\mathrm{He})=11.30$ (Fig. 3). The three lines for which we were not able to obtain a good synthetic profile are the HeI $\lambda \lambda 5876,6678$ and $7065 \AA$. The derived helium abundance seems to be larger than the solar value, however the difficulty in converting the observed equivalent widths of helium lines to abundances is a well known problem since the work of Auer \& Mihalas (1972). Particularly, Gies \& Lambert (1992) found that in stars with effective temperature larger than $30000 \mathrm{~K}$, helium appears to be overabundant with respect to the Sun.

To overcome the difficulties resulting from the spectral modeling, we compared our measurements of equivalent widths with the values reported by Gies \& Lambert (1992) for stars with similar effective temperature and gravity (Table 2). The equivalent widths we measured for the HeI lines are only slightly larger than those derived by Gies \& Lambert (1992) and this discrepancy does not seem significant enough to conclude that HD 207538 is a helium peculiar star. The situation of the HeII $\lambda 4686 \AA$ line is more complicated since some kind of erratic behavior of the equivalent width versus effective temperature has been reported. In any case, the mean value of the helium abundance calculated by Gies \& Lambert in the
Table 2. Equivalent widths of the helium lines measured with the SARG-TNG and at Catania Astrophysical Observatory (last two columns). We compared our measurements with the B-type stars of similar effective temperature extracted from Gies \& Lambert (1992). All equivalent widths are expressed in $\mathrm{m} \AA$.

\begin{tabular}{l|ccccc|cc}
\hline \hline & $\mathrm{A}$ & $\mathrm{B}$ & $\mathrm{C}$ & $\mathrm{D}$ & $\mathrm{E}$ & \multicolumn{2}{c}{ HD 207538 } \\
HeII & & & & & & SARG-TNG & Catania \\
\hline 4686 & 206 & 192 & 747 & 602 & 858 & $500 \pm 60$ & \\
5411 & & & & & & $203 \pm 34$ & $250 \pm 40$ \\
\hline \hline HeI & & & & & & & \\
\hline 4713 & 251 & 246 & 243 & 230 & 235 & $297 \pm 28$ & \\
4921 & & & & & & $514 \pm 44$ & $580 \pm 40$ \\
5015 & 260 & 268 & 262 & 258 & 268 & $309 \pm 20$ & $308 \pm 21$ \\
5047 & 139 & 144 & 114 & 130 & 102 & $142 \pm 19$ & $176 \pm 20$ \\
5876 & & & & & & $825 \pm 29$ & \\
6678 & & & & & & $714 \pm 33$ & \\
7065 & & & & & & $637 \pm 54$ & \\
\hline
\end{tabular}

A - HD 34816, $T_{\text {eff }}=29890 \mathrm{~K}, \log g=4.22, \xi=6.7 \mathrm{~km} \mathrm{~s}^{-1}$. B - HD 36960, $T_{\text {eff }}=29960 \mathrm{~K}, \log g=4.30, \xi=4.3 \mathrm{~km} \mathrm{~s}^{-1}$. C - HD 34078, $T_{\text {eff }}=31420 \mathrm{~K}, \log g=4.07, \xi=6.9 \mathrm{~km} \mathrm{~s}^{-1}$. D - HD 36512, $T_{\text {eff }}=32470 \mathrm{~K}, \log g=4.29, \xi=5.7 \mathrm{~km} \mathrm{~s}^{-1}$. E - HD 214680, $T_{\text {eff }}=34370 \mathrm{~K}, \log g=4.29, \xi=12.2 \mathrm{~km} \mathrm{~s}^{-1}$.

LTE approximation for these stars is $\log \epsilon(\mathrm{He})=11.29 \pm 0.05$, a value consistent with our estimation, within errors.

To verify if spectral variability exists, we measured the equivalent width of the helium lines present in our two visible spectra. The equivalent widths of these lines obtained with both telescopes were reported in Table 2. According to our measurements and the quoted literature studies no helium spectroscopic variability has been detected, confirming the non peculiar nature of HD 207538.

\subsection{IUE data}

In an attempt to reproduce the spectrum recorded by $I U E$, we computed the photospheric spectrum with the same parameters used for the optical data, after broadening it by the rotational velocity and by the instrumental resolving power of $R=7500$, corresponding at $1500 \AA$ to the nominal resolution $\Delta \lambda \sim 0.2 \AA$.

In Fig. 4 we show the comparison between the synthetic spectrum and the observations in three intervals. With a few exceptions, most of the spectral lines are quite well reproduced.

In the $I U E$ range we identified one NiIV line at $\lambda 1398.193 \AA$ and two MnIV lines at $\lambda 1664.772 \AA$ and $\lambda 1666.981 \AA$. The former has been reproduced with an underabundance of $0.45 \mathrm{dex}$ with respect to the standard value, the latter on the contrary are compatible with a normal abundance. Noteworthy is the fact that the HeII $\lambda 1640 \AA$ line is well reproduced with the helium abundance computed in the previous section. Other lines, such as those generated by iron, silicon and zinc, have been modeled using the photosperic abundances computed for the FUSE spectrum (see next section). As to carbon and aluminum, we used the abundances computed in the previous section for the SARG-TNG data. 


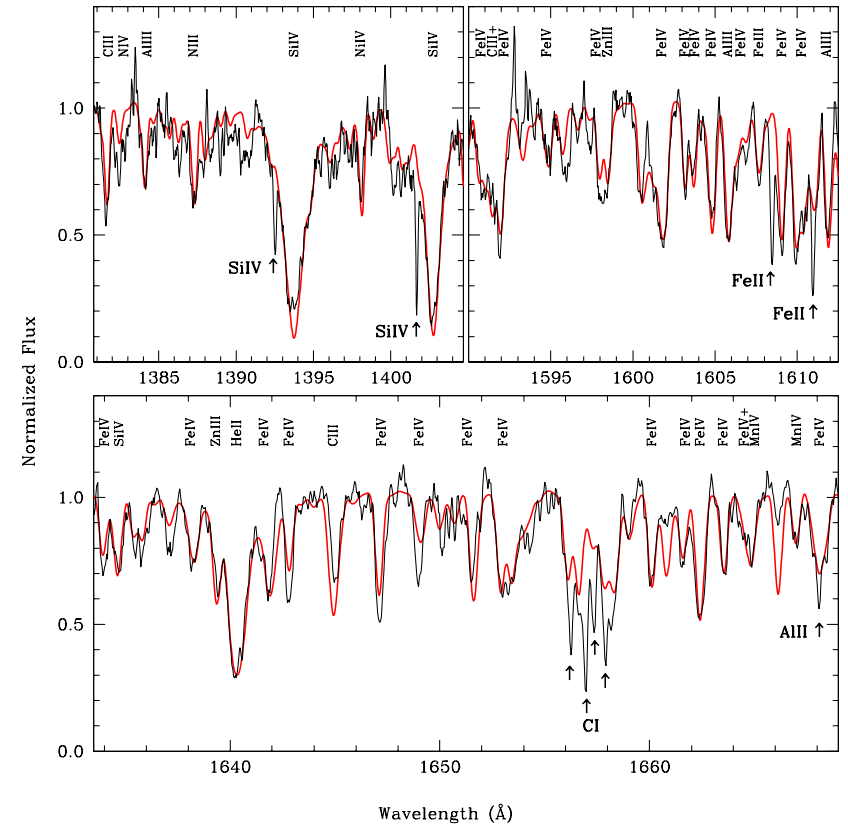

Fig. 4. Comparison between theoretical and observed spectra in the IUE range selected $(\lambda \lambda 1380-1670) \AA$. In each panel we label the identified lines. ISM lines have been marked with arrows.

A contamination due to interstellar matter is present in these intervals. In Fig. 4 we marked with arrows the lines we identified using the list of ISM lines that occur in the IUE range published by St-Louis \& Smith (1991).

IUE provides us with further evidence of the absence of variability as no differences exist between the flux of spectra obtained at different dates (see Sect. 3.2).

\subsection{FUSE data}

To compute the synthetic spectrum in this wavelength region, we used the abundances inferred from the $S A R G-T N G$ data for those elements present in both intervals, and we determined the abundance for those elements whose lines are not present in the optical region, by matching the profiles observed in the FUV range.

To take into account the large number of interstellar lines the computed photospheric spectrum has been multiplied by the ISM model (see next section) and then compared with the observation. Solar standard abundances were found compatible, within the experimental errors, with the line profiles observed for CNO elements and for SiIII, SiIV, PIV, PV and Siv. On the other hand, underabundances were estimated for iron and zinc. Standard abundances predict the presence of a Cliv line at $\lambda 1123.785 \AA$, not observed in our data. In Figs. 7 and 8 we show the comparison between the synthetic and the observed spectrum.

Inferred abundances are reported in Table 3 where they are compared with those calculated by Daflon et al. (1999), Gies \& Lambert (1992) and the Sun (Grevesse et al. 1996).

Departures from LTE can affect the inferred chemical abundances. The magnitude of non-LTE corrections has been investigated by Daflon et al. (1999) for a sample of stars in Cep OB2
Table 3. Abundances derived for HD 207538 expressed in the usual form $\log \epsilon(\mathrm{El})$ compared with those estimated by Daflon et al. (1999) (Col. 3), by Gies \& Lambert (1992) (Col. 4) and the Sun (Grevesse et al. 1996, Col. 5). Errors have been estimated to be 0.2 dex.

\begin{tabular}{lcccc}
\hline \hline El & $\begin{array}{c}\text { This } \\
\text { work }\end{array}$ & D & G \& L & Sun \\
\hline $\mathrm{He}$ & 11.30 & & $11.29 \pm 0.05^{\mathrm{a}}$ & $10.99 \pm 0.03$ \\
$\mathrm{C}$ & 8.50 & & $8.40 \pm 0.11^{\mathrm{a}}$ & $8.55 \pm 0.05^{\mathrm{b}}$ \\
$\mathrm{N}$ & 8.11 & $7.99 \pm 0.16$ & $7.70 \pm 0.06^{\mathrm{a}}$ & $7.97 \pm 0.07$ \\
$\mathrm{O}$ & 8.66 & $8.67 \pm 0.16$ & $8.78 \pm 0.12^{\mathrm{a}}$ & $8.88 \pm 0.07^{\mathrm{b}}$ \\
$\mathrm{Al}$ & 6.53 & & $6.62 \pm 0.20^{\mathrm{a}}$ & $6.47 \pm 0.07$ \\
$\mathrm{Si}$ & 7.61 & $7.74 \pm 0.05$ & $7.35 \pm 0.02^{\mathrm{a}}$ & $7.55 \pm 0.05$ \\
$\mathrm{P}$ & 5.51 & & & $5.45 \pm 0.04$ \\
$\mathrm{~S}$ & 7.27 & & $7.21 \pm 0.12$ & $7.33 \pm 0.11$ \\
$\mathrm{Fe}$ & 7.10 & & $7.71 \pm 0.23$ & $7.50 \pm 0.04$ \\
$\mathrm{Mn}$ & 5.45 & & & $5.39 \pm 0.03$ \\
$\mathrm{Ni}$ & 5.80 & & & $6.25 \pm 0.01$ \\
$\mathrm{Zn}$ & 4.20 & & & $4.50 \pm 0.08$ \\
\hline
\end{tabular}

${ }^{a}$ Average value calculated over the abundances reported for the five stars quoted in Table 2.

${ }^{b}$ According to a recent revision of the carbon and oxygen abundances in the Sun performed by Allende Prieto et al. (2002), these values should be $8.39 \pm 0.04$ and $8.69 \pm 0.07$ respectively. They are in better agreement with the $\mathrm{B}$ star abundances.

association. They found that these corrections are generally of the order of 0.1 dex or even smaller. In particular, for carbon and oxygen they found the corrections are negligible.

\section{Interstellar spectrum analysis}

The ISM analysis was conducted with the profile fitting software OWENS described in Hébrard et al. (2002). This software allows us to perform multiple fits simultaneously combining several spectral windows and several species. By combining different species with different masses, this procedure makes it possible to have a good handle on the interstellar thermal and turbulent broadenings along the sightline. With the help of the stellar model, we first selected in the spectrum the ISM absorption lines which were not blended with any known stellar features. The $f$-values used for FeII are the revised from Howk et al. (2000) and, for all the other atomic species, the $f$-values are from Morton (1991) and Welty (1999). We adopt wavelengths, oscillator strengths, and damping constants for the molecular hydrogen transitions from Abgrall et al. (1993) for the Lyman system and from Abgrall et al. (1993) for the Werner system.

\subsection{Velocity structure}

A detailed interstellar velocity structure was inferred from visible NaI data at high resolution $(R \approx 115000)$. Fits to the NaI line profiles indicate at least 3 components as shown in Fig. 5. The two weaker components are clearly separated at the resolution of the instrument and centered at $-14 \mathrm{~km} \mathrm{~s}^{-1}$ and $-19 \mathrm{~km} \mathrm{~s}^{-1}$ relative to the dominant component with respective Doppler broadenings (or $b$-values) of 3 and $2 \mathrm{~km} \mathrm{~s}^{-1}$. The total column density for these two components is of the order of a 


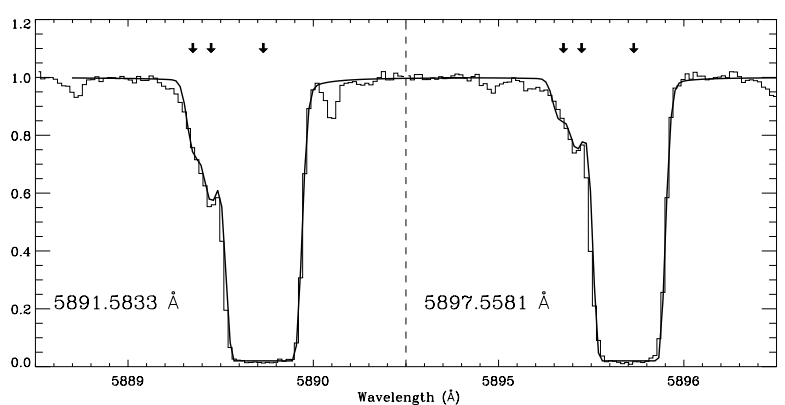

Fig. 5. Fits of the NaI absorption lines. Three components are detected, the central components represents more that $99 \%$ of the total column density: $5.3 \times 10^{14} \mathrm{~cm}^{-2}$.

few times $10^{12} \mathrm{~cm}^{-2}$ which represents only $1 \%$ of the total NaI column. The strong saturation of the dominant NaI component and the low resolution of the FUSE data do not allow us to determine its velocity structure unambiguously. High-resolution data are required to accurately determine the total number of components present along the sightline.

Lacour et al. (2003) reported four $\mathrm{CH}$ components along this sightline coinciding with the core of the strong NaI absorption. Additional components are however necessary to fit the NaI profile. Subsequently, in order to account for potential saturation effects due to narrow components we considered a total of 6 components in our velocity structure: 4 components associated with the $\mathrm{CH}$ absorptions and 2 additional atomic components as seen in the NaI profile. Note however that other hidden atomic components might still be present in the core of the NaI escaping detection due to insufficient resolution.

\subsection{Atomic species}

We performed a six-component profile fitting for the atomic species seen in absorption: FeII, PII, OI, NI, and ArI. Because most of these atomic lines are saturated and because of the moderate resolution of the FUSE spectrograph $\left(\approx 20 \mathrm{~km} \mathrm{~s}^{-1}\right)$, it is not possible to retrieve the information on the velocity structure from the FUSE data itself. Thus, the velocities of each component are constrained as described in the previous subsection and are not free parameters in the fit. In particular, the relative ratios of the four stronger components were fixed to values derived from the high-resolution $\mathrm{CH}$ absorption line profiles. The fitting was performed simultaneously over 22 spectral FUSE windows. Results are shown in Fig. 6 and Table 4.

The $\mathrm{Ly}_{\beta} \mathrm{HI}$ line is too severely blended by molecular hydrogen to be of any help in determining the total HI column density. Moreover, the $\mathrm{Ly}_{\beta}$ HI line is saturated and therefore does not constitute a sensitive probe for the column density. Thus we simply report here the value derived by Rachford et al. (2002) (see Table 4) using a canonic relation between the extinction, $\mathrm{H}_{2}$, and $\mathrm{HI}$. The resulting profile seems to adequately fit our data especially in the 1026 and $1030 \AA$ windows.

Our determination of the interstellar oxygen abundance is based on the profile of the $1039 \AA$ line. The $\mathrm{O} / \mathrm{H}$ ratio of $240_{-100}^{+200} \mathrm{ppm}$ that we derive is consistent within $1 \sigma$ with the newly revised interstellar oxygen gas phase abundance

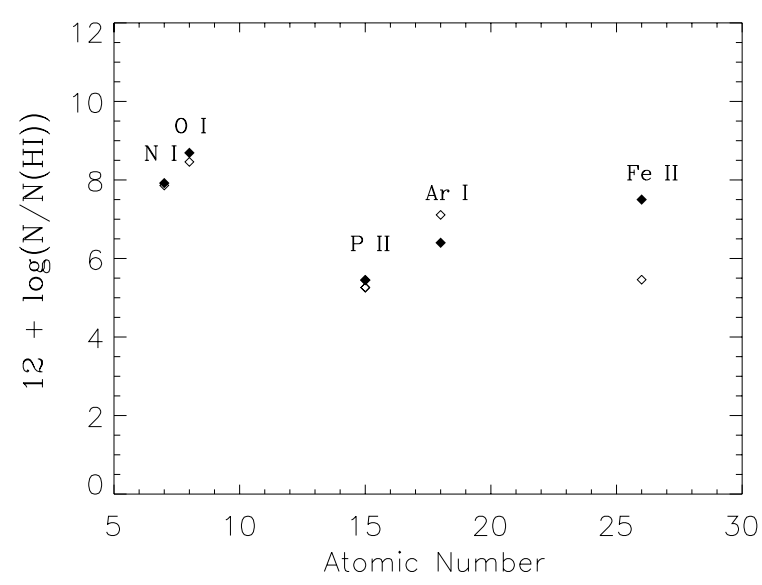

Fig. 6. Comparison of elemental abundances with respect to hydrogen in the standard logarithmic scale (Grevesse \& Sauval 1998). Open diamonds represent the measurements toward HD207538 and black diamonds represent the solar references.

(André et al. 2003) of 408 ppm. However, the fact that our mean value lies well below the adopted interstellar abundance likely indicates a larger depletion along this sightline. Such enhanced depletion has been reported by Cartlege et al. (2001) toward reddened stars with $E_{(B-V)}$ similar to HD 207538.

The nitrogen column density was derived from the $1134 \AA$ triplet. It is usually known that NI is not depleted onto grains and we indeed find a $\mathrm{N} / \mathrm{H}$ ratio close to the solar value of $83 \mathrm{ppm}$ (Grevesse \& Sauval 1998) although with very large error bars: $\mathrm{N} / \mathrm{H}=70_{-30}^{+210} \mathrm{ppm}$.

As expected for a refractory element, iron is found to be depleted by about 2 dex compared to the solar value which is a further hint toward a large grain population along this sightline. Noteworthy is the FeII column density which is well constrained by the use of the relatively weak transition at $1127 \AA$.

Being a noble gas, ArI is not expected to be severely depleted onto grains. But based on the $1048 \AA$ line, we derive a surprising super solar abundance for ArI. This high value may well be due to the blending of the interstellar line with an unpredicted stellar line.

The case of ClI deserves a particular attention for it is not expected to arise in the diffuse atomic components but in gas components with optically thick molecular hydrogen lines (Jura 1974). It is therefore expected that ClI coexists with the molecular content along the sightline. We therefore used the velocity structure derived from the molecular components $(\mathrm{CH})$ to fit the few Cli lines for which accurate $f$-values were measured.

It should be noted that some $\mathrm{CI}, \mathrm{CI}^{*}$ and $\mathrm{CI}^{* *}$ lines are also detected between $1100 \AA$ and $1120 \AA$ but the transitions are blends of multiplets and without high resolution STIS data we are unable to disentangle the various excited states (Sonnentrucker et al. 2002). Furthermore, due to the severe stellar blending over so many absorption lines we cannot produce a reliable $\mathrm{CI}, \mathrm{CI}^{*}$ and $\mathrm{CI}^{* *}$ model. Such a model would be helpful to derive the average density. Finally, we point out that the presence of large quantities of molecular hydrogen did not allow us to further investigate other atomic species in this spectrum. 


\subsection{Molecular species}

Below $1100 \AA$, the $\mathrm{H}_{2}$ lines are the most prominent features seen in absorption. The analysis of the column density of the $J=0,1$ rotational levels has already been performed by Rachford et al. (2002). They showed that this line of sight may contain translucent cloud material and that some of the molecular hydrogen content originates in several more diffuse components. As mentioned before, four components are seen in $\mathrm{CH}$, all of them well within $10 \mathrm{~km} \mathrm{~s}^{-1}$ which means that they cannot be resolved by FUSE (Lacour et al. 2003).

Adopting this velocity structure, we performed a global profile fitting of the $J$ levels up to $J=7$. Our results agree with those of Rachford et al. (2002) for $J=0,1$ and we find a temperature $T_{01}$ of $70 \mathrm{~K}$. Because the profiles of the higher $J$ levels are saturated, they are not sensitive to the column density and we could only measure lower limits to their column densities. Note also that $J$ levels higher than $J=1$ contribute a negligible fraction to the total $\mathrm{H}_{2}$ column density (see Table 4). We hence found a total $\mathrm{H}_{2}$ column density of $8.2 \times 10^{20} \mathrm{~cm}^{-2}$ along the sightline toward HD 207538.

An analysis of the $1076 \AA \mathrm{CO}(J=0)$ band was also performed. Because this line arises close to a strong $\mathrm{H}_{2}$ absorption and because the $\mathrm{CO}$ band is heavily saturated, we can only report a stringent lower limit to its column density of $10^{15} \mathrm{~cm}^{-2}$, leading to a conversion factor of $1.2 \times 10^{-6}$. This CO-to- $\mathrm{H}_{2}$ conversion factor is consistent with the range expected when translucent material is present along a sightline (van Dishoeck $\&$ Black 1980). Our result therefore further suggests that along this particular line of sight some denser, colder gas is present.

HD absorption lines are also seen along this sightline. Their study is discussed in Lacour et al. (2003). They find that a $\mathrm{HD} / \mathrm{H}_{2}$ ratio of $8.9 \times 10^{-6}$ cannot be explained unless the main molecular component is dense enough to shield the HD molecule from the external UV radiation field. The recent chemical model of translucent clouds of Le Petit et al. (2002) applied to this sightline indicates a molecular fraction of at least $80 \%$ and number densities of the order of a few hundred atoms per cubic centimeters, also consistent with the idea that some translucent material is present in the interstellar gas.

\section{Conclusions}

We performed a detailed abundance analysis of both the stellar atmosphere and the interstellar medium toward the suspected CP star HD 207538. We combined Far-UV spectra obtained by FUSE, UV spectra obtained by IUE with our optical spectra obtained with the ground-based SARG-TNG facility.

Synthetic spectra were computed using ATLAS9 and SYNTHE for the stellar contribution and by the OWENS code for the interstellar medium spectrum. Effective temperature and surface gravity were fixed by matching the UV and visible flux distributions. We found that our values are in agreement with the values given by Daflon et al. (1999). Microturbulent velocity was determined assuming that the oxygen abundance inferred from ten lines is independent of the measured equivalent width. Rotational velocity was determined by matching the profiles of five unblended metal lines.
Our model underestimates the observed flux only in the spectral range 1110-1170 $\AA$; this discrepancy could be ascribed to various causes as we discussed at the end of Sect. 4.2.

Regarding the abundances in CP stars, usually helium is underabundant in cool CP stars and overabundant in hot CP stars. Carbon, oxygen, nitrogen and magnesium are underabundant and iron peak elements are overabundant. In our study, with the exception of the underabundance found for iron, nickel and zinc, the other metals show lines profiles compatible with the standard solar chemical composition (Grevesse et al. 1996). We also find that the helium abundance is $\log \epsilon(\mathrm{He})=11.30$, value compatible with the standard value measured for B-type stars reported by Gies \& Lambert (1992). Therefore, our results seem to indicate that HD 207538 does not have peculiar abundances.

Regarding the main observational fingerprint of the $\mathrm{CP}$ stars, namely light, spectral and magnetic variability: $a$ ) we did not find any evidence of variability in the equivalent widths of helium lines detected in both of our datasets and in the various literature contributions, $b$ ) Vetö et al. (1980) found the light curve constant and we confirm the absence of variability from HIPPARCOS data, $c$ ) no magnetic measurements were reported so far, unfortunately. All this evidence leads us to conclude that HD 207538 has been incorrectly reported in the Renson (1991) catalogue.

In conclusion, based on Kurucz codes our stellar analysis shows that we were able to reproduce the principal spectral features observed in HD 207538 from the far-UV to visible range using a single set of atmospheric parameters $\left(T_{\text {eff }}, \log g\right.$ and $\left.\xi\right)$ and chemical abundances.

The analysis of the interstellar gas content in the FUSE range toward $\mathrm{HD} 207538$ led to estimates of the $\mathrm{H}_{2}, \mathrm{CO}$, NI, OI, ClI, ArI, FeII and PII column densities. The high CO-to- $\mathrm{H}_{2}$ conversion factor, the large molecular fraction as derived from the $\mathrm{HD} / \mathrm{H}_{2}$ ratio both argue for the existence of some translucent

Table 4. ISM molecular and atomic column densities toward HD207538.

\begin{tabular}{cc}
\hline \hline Species & $\log N($ dex $)$ \\
\hline ArI & $16.45_{-.50}^{+.25}$ \\
CO & $>15.00$ \\
Cl I & $14.4_{-.90}^{+.80}$ \\
Fe II & $14.80_{-.10}^{+.10}$ \\
H I & $21.34 \pm 0.12$ \\
H20 & $20.64 \pm 0.07$ \\
H21 & $20.58 \pm 0.05$ \\
H22 & $18.75_{-.15}^{+.10}$ \\
H23 & $17.95_{-.20}^{+.25}$ \\
H24 & $17.60_{-.60}^{+.40}$ \\
H25 & $15.95_{-2.0}^{+1.05}$ \\
H26 & $>14.00$ \\
H27 & $>14.00$ \\
HD & $15.86 \pm 0.35$ \\
N I & $17.2 \pm 0.5$ \\
Na I & $>14.72$ \\
O I & $17.80_{-.35}^{+.35}$ \\
P II & $13.00 \ll 14.60$ \\
\hline
\end{tabular}



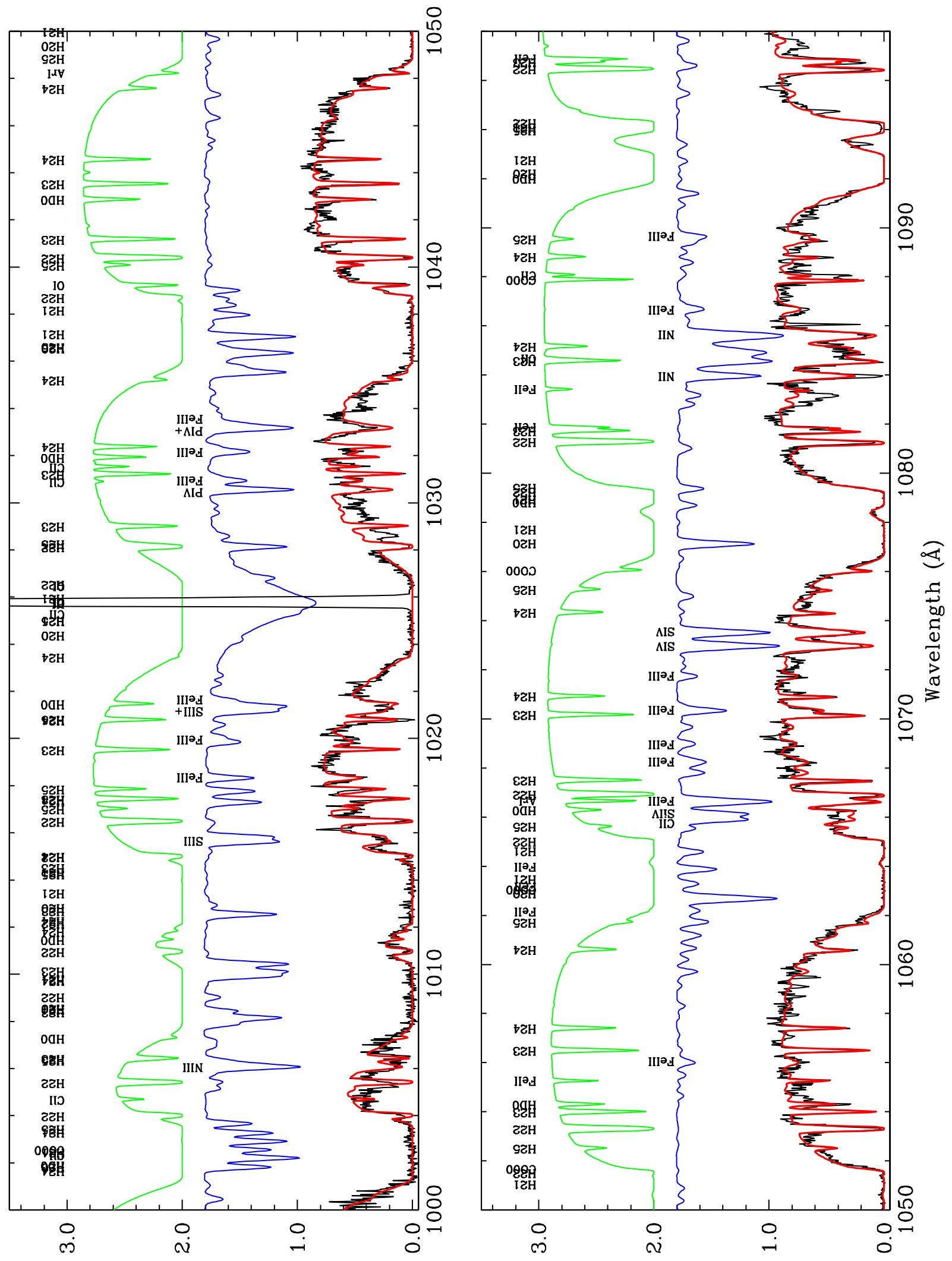

xnIJ pəz!̣euison

Fig. 7. Comparison between theoretical and observed spectra in the FUV range $\lambda 1000-1098 \AA$. In each box we have: at the top the ISM model with labelled the identified lines, at the middle the synthetic photospheric model with the identified lines and at the bottom, the observed spectrum compared with the total model. 


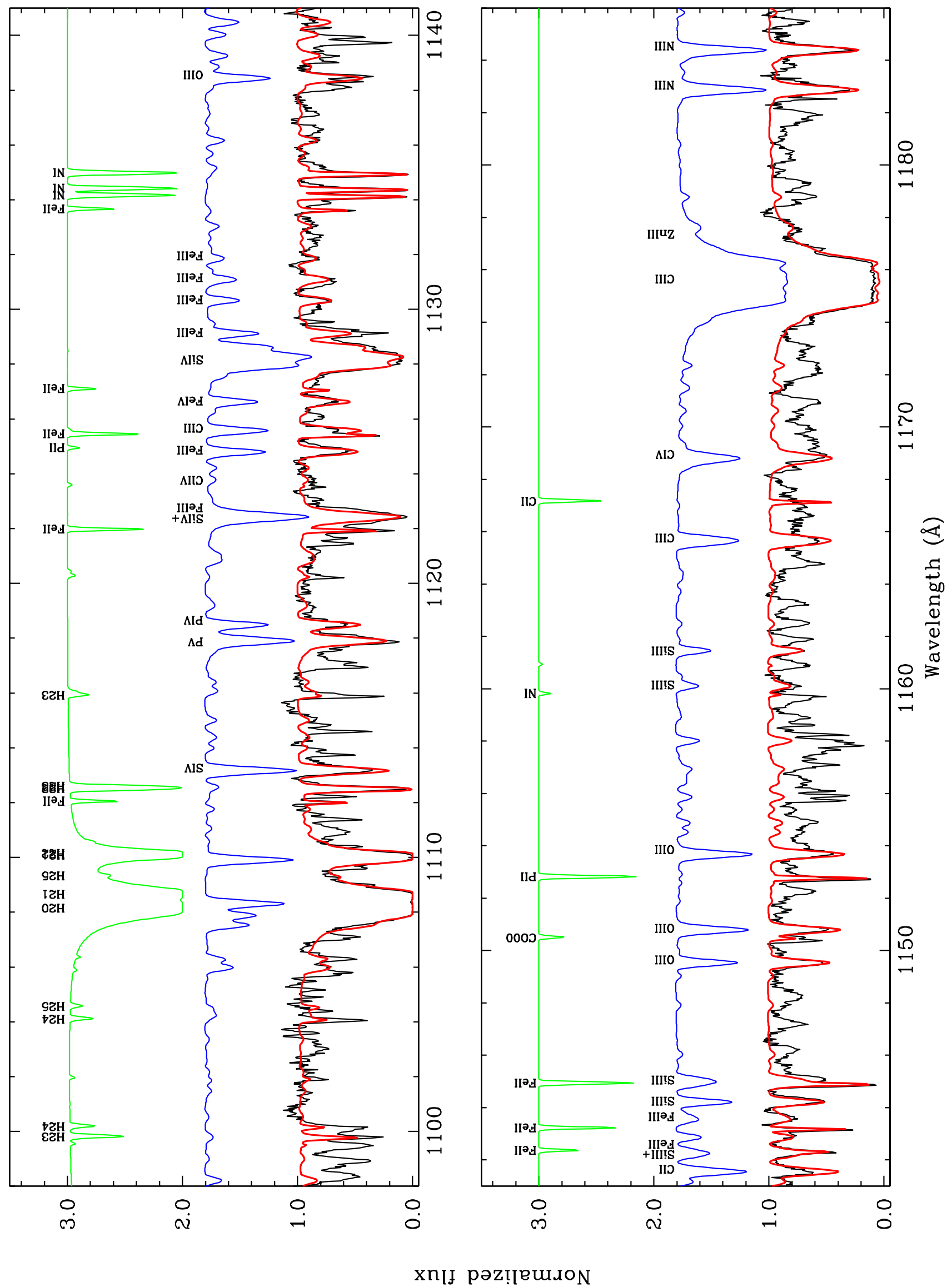

Fig. 8. Comparison between theoretical and observed spectra in the FUV range $\lambda 1098-1188 \AA$ A. Symbols as in Fig. 7. 
material among the diffuse interstellar gas components toward HD 207538. However, high resolution observations of atomic species such as $\mathrm{CI}, \mathrm{CI}^{*}$ and $\mathrm{CI}^{* *}$ using the STIS range are required to confirm the existence of denser cloud components and to study the depletion pattern along this sightline.

Acknowledgements. This work is based on observations made with the NASA-CNES-CSA Far Ultraviolet Spectroscopic Explorer (FUSE) which is operated for NASA by the "The Johns Hopkins University" under NASA contract NAS5-32985, on observations made with the Italian Telescopio Nazionale Galileo (TNG) operated on the island of La Palma by the Centro Galileo Galilei of the INAF (Istituto Nazionale di Astrofisica) at the Spanish Observatorio del Roque de los Muchachos of the Instituto de Astrofisica de Canarias and on INES data from the International Ultraviolet Explorer (IUE) satellite.

This work has made use of the profile fitting procedure OWENS developed by M. Lemoine and the FUSE French Team.

This research has made use of the SIMBAD database, operated at CDS, Strasburg, France.

We wish to thank the referee, Dr. P. Barklem, for his help in improving the manuscript.

\section{References}

Abgrall, H., Roueff, E., Launay, F., Roncin, J. Y., \& Subtil, J. L. 1993, A\&AS, 101, 273

Abgrall, H., Roueff, E., Launay, F., Roncin, J. Y., \& Subtil, J. L. 1993b, A\&AS, 101, 323

Allende Prietro, C., Lambert, D. L., \& Asplund, M. 2002, ApJ

André, M. K., et al. 2003, in preparation

Auer, L., \& Mihalals, D. 1972, ApJS, 24, 193

Buss, R. H., Kruk, J. W., \& Ferguson, H. C. 1995, ApJ, 454, L55

Cardelli, J. A., Clayton, G. C., \& Mathis, J. S. 1989, ApJ, 345, 245

Cartledge, S. I. B., Meyer, D. M., Lauroesch, J. T., \& Sofia, U. J. 2001, ApJ, 562, 394

Castelli, F., \& Kurucz, R. L. 1994, A\&A, 281, 817

Chavez, M., Stalio, R., \& Holberg, J. B. 1995, ApJ, 449, 280

Conti, P. S. 1974, ApJ, 187, 539

Conti, P. S. 1973, ApJ, 179, 161

Conti, P. S., \& Alschuler, W. R. 1971, ApJ, 170, 325

Daflon, S., Cunha, D., Becker, S. R., \& Smith, V. V. 2001, ApJ, 552, 309

Daflon, S., Cunha, K., \& Becker, S. R. 1999, ApJ, 522, 950
De Zeeuw, P. T., Hoogerwerf, R., De Bruijne, J. H. J., et al. 1999, AJ, 117,354

Dixon, W. V. D., \& Hurwitz, M. 1998, A\&A, 500, L29

Frémat, Y., Zorec, J., Hubert, A.-M., et al. 2002, A\&A, 385, 986

Gies, D. R., \& Lambert, D. L. 1992, ApJ, 387, 673

Gratton, R., Bonanno, G., Bruno, P., et al. 2003, Ap\&SS (in press)

Grevesse, N., \& Sauval, A. J. 1998, Space Sci. Rev., 85, 161

Grevesse, N., Noels, A., \& Sauval, A. J. 1996, in Cosmic Abundances, ed. S. S. Holt, \& G. Sonneborn, ASP Conf. Ser., p. 117

Grigsby, J. A., Morrison, N. D., \& Anderson L. S. 1992, ApJS, 78, 205

Hébrard, G., Lemoine, M., Vidal-Madjar, A., et al. 2002, ApJS, 140, 103

Howk, J. C., Sembach, K. R., Roth, K. C., \& Kruk, J. W. 2000, ApJ, 544, 867

Jura, M. 1974, ApJ, 190, L33

Kurucz, R. L. 1993, A new opacity-sampling model atmosphere program for arbitrary abundances, in ed. M. M. Dworetsky, F. Castelli, \& R. Faraggiana, IAU Col. 138, Peculiar versus normal phenomena in A-type and related stars, ASP Conf. Ser., 44, 87

Kurucz, R. L., \& Avrett, E. H. 1981, SAO Special Rep., 391

Lacour, S., Le Petit, F., Sonnentrucker, P., et al. 2003, in preparation

Leckrone, D. S., Fowler, J. W., \& Adelman, S. J. 1974, A\&A, 32, 237

Leone, F., \& Manfrè, M. 1996, A\&A, 315, 526

Le Petit, F., Roueff, E., \& Le Bourlot, J. 2002, A\&A, 390, 369

Mathys, G. 1989, A\&AS, 81, 237

Moos, H. W., Cash, W. C., Cowie, L. L., et al. 2000, ApJ, 538, L1

Morales, C., Orozco, V., Gómez, J. F., et al. 2001, ApJ, 522, 278

Morton, D. C. 1991, ApJS, 77, 119

Nichols, J. S., \& Linsky, J. L. 1996, AJ, 111, 517

Penny, L. 1996, ApJ, 463, 737

Peterson, B. A. 1966, ApJ, 145, 735

Rachford, B. L., Snow, T. P., Tumlinson, J., et al. 2002, ApJ, 577, 221

Renson, P., Catalogue Général des Etoiles Ap et Am, Institut d'Astrophysique, Université de Liège

Sahnow, D. J., Moos, H. W., Ake, T. B., et al. 2000, ApJ, 538, L7

Sonnentrucker, P., Friedman, S. D., Welty, D. E., York, D. G., \& Snow, T. P. 2002, ApJ, 576, 241

Snow, T. P., Rachford, B. L., \& Figoski, L. 2002, ApJ, 573, 662

Stibbs, D. W. N. 1950, MNRAS, 110, 395

St-Louis, N., \& Smith, L. J. 1991, A\&A, 252, 781

Stellingwerf, R. F. 1978, ApJ, 224, 953

van Dishoeck, E. F., \& Black, J. H. 1989, ApJ, 340, 273

Vetö, B., Schoeneich, W., \& Rustamov, Iu. S. 1980, AN, 301, 317

Welty, D. E., Hobbs, L. M., \& Lauroesch, J. T., et al. 1999, ApJS, 124, 465 